\title{
Prevalência de Cervicalgia em Acadêmicos de Odontologia de um Centro Universitário
}

\author{
Prevalence of Cervical Pain in Dental Student of a University Center
}

Prevalencia de la Cervicalgia en Académicos del Odontología en un Centro Universitario

Adryelle de Farias Silva ${ }^{1}$

Mariana Alves Lima da Costa ${ }^{2}$

Renata Sampaio Rodrigues Soutinho ${ }^{3}$

Alexsandra de Souza Pedrosa ${ }^{4}$

\section{Resumo}

Objetivo: Verificar a prevalência de cervicalgia em estudantes de odontologia de um Centro Universitário. Método: Tratou-se de um estudo observacional, analítico de caráter transversal, realizado no período de janeiro a junho de 2015 em uma Clinica Escola de Odontologia de um Centro Universitário da cidade de Maceió -AL, com uma amostra de 67 acadêmicos em estágio supervisionado, onde foi utilizado um formulário de coleta de dados e o Indice de
Incapacidade Relacionada ao Pescoço. A análise estatística foi do tipo descritiva e inferencial utilizando-se o teste qui-quadrado. Resultados: A prevalência de cervicalgia, foi de $44,8 \%$, não tendo sido observado diferenças significativas entre a queixa e as variáveis sexo, estado civil, atividade física, período acadêmico, número de práticas e área do estágio. Em relação a interferência da dor nas atividades cotidianas, a maioria, apresentou incapacidade leve. Conclusão: Pode-se observar que a prevalência de cervicalgia encontrada

${ }^{1}$ Fisioterapeuta. Graduada em Fisioterapia pelo Centro Universitário CESMAC. Autora correspondente: Avenida Tiradentes, 302, Pratinhas. CEP: 57360-00. Girau do Ponciano - Alagoas, Brasil. E-mail: pollydryca@hotmail.com

${ }^{2}$ Fisioterapeuta. Graduada em Fisioterapia pelo Centro Universitário CESMAC.

${ }^{3}$ Fisioterapeuta. Mestre em Pesquisa em Saúde pelo Centro Universitário CESMAC. Especialista em Urologia e Obstetrícia pela Maurício de Nassau e em Gestão em Saúde pela UFAL.

${ }^{4}$ Fisioterapeuta. Doutoranda em Ciências pelo Programa de Medicina Interna e Terapêutica - DINTER UNIFESP/ UNCISAL. Mestre em Fisioterapia pela Universidade Cidade de São Paulo- UNICID. Especialista em Fisioterapia Traumato-Ortopédica pela Universidade Gama Filho.

Recebido: Mar/2017 - Aceito: Jul/2017. 
Silva AF, Costa MAL, Soutinho RSR, Pedrosa AS 423

Prevalência de Cervicalgia em Acadêmicos de Odontologia

entre os acadêmicos de odontologia, ainda no período de graduação, enquadra-se nos valores propostos pela literatura em profissionais da área, o que reforça o fato de que devem ser elaboradas estratégias de prevenção desde a época da graduação, a fim de minimizar o impacto desta durante a vida profissional.

\section{Descritores: Cervicalgia; Estudantes de Odontologia; Prevalência; Dor.}

\section{Abstract}

Objective: To determine the prevalence of cervical pain in dental students from a University Center. Methods: This was an observational, analytical transverse character, performed in the period January-June 2015 the Clinical University Center School of Dentistry Cesmac, with a sample of 67 students in supervised training where we used a data collection form and the Disability Index Related to Neck. The statistical analysis was descriptive and inferential type using the chi-square test. Results:

The prevalence of cervical pain was $44.8 \%$, and had no significant differences between the complaint and the variables sex, marital status, physical activity, academic period, number of practical and stage area.
Regarding the interference of pain on daily activities, most had mild disability. Conclusion: It can be observed that the prevalence of cervical pain found among dental students, even the graduation period, is part of the values proposed in the literature for professionals, which reinforces the fact that should be developed prevention strategies from the time of graduation, in order to minimize the impact of this during working life.

Descriptors: Neck pain; Students Dental; Prevalence; Pain.

\section{Resumen}

Objetivos: Verificar la prevalencia de la cervicalgia en estudiantes del odontología de un Centro Universitario. Métodos: Se trata de un estudio observacional, analítico de carácter trasversal, realizado en el periodo de enero a junio de 2015 en la Clínica Escuela de Odontologia del Centro Universitario Cesmac, con una muestra de 67 académicos en las prácticas clínicas, se ha aplicado un formulario para recoger los datos y el Índice de Incapacidad Relacionada del Cuello. El análisis estadística ha sido del tipo descriptiva e inferencial, se utilizando del test qui-quadrado. Resultados: La 
prevalencia de la cervicalgia de 44\%, no se ha observado diferencias significativas entre las quejas y las variaciones del sexo, estado civil, actividad fisica, ciclo de la carrera, número de prácticas clínicas y el área de actuación de las prácticas. Con relación la interferencia del dolor en las actividades cotidianas, la mayoría, presento la incapacidad leve. Conclusión: La prevalencia de la cervicalgia encontrada en los académicos del odontología, aunque en el ciclo de la carrera, se encuadra en los valores propuestos por la literatura en profesionales de la área, lo que refuerza el fato de que se debe crear estrategias para la prevención desde las prácticas de la graduación, a fin de, minimizar el impacto de esta, durante la vida profesional.

\section{Descriptores: Cervicalgia; Estudiantes de Odontología; Prevalencia; Dolor.}

\section{Introdução}

A cervicalgia se caracteriza por uma dor localizada na coluna cervical proveniente de distúrbios musculoesqueléticos em região posterior do pescoço e superior das escápulas ou zona dorsal alta, isenta de sinais de radiculopatias ou seja, uma dor que não apresenta irradiação ou parestesia $^{(1-2)}$.

Pode ser classificada em aguda, quando há presença de dor súbita; ou crônica, quando torna-se persistente. Quanto à etiologia, pode ser considerada idiopática, quando não possui causa aparente ou ser uma dor decorrente de um trauma ${ }^{(3-4)}$.

Apesar da dor ser o sintoma mais evidente e incomodo, a cervicalgia pode estar associada a limitação de movimentos, quadros inflamatórios,danos aos tecidos periarticulares, fadigabilidade de flexores e extensores da cervical, redução da propriocepção e rigidez local $^{(4-5)}$.

A dor em região cervical é uma lesão osteomuscular de grande importância na sociedade mundial, caracterizando assim um problema de ordem de saúde pública, pois acaba afetando a qualidade de vida e a saúde da sociedade de forma global, levando muitos indivíduos a quadros depressivos, dependência medicamentosa, labilidade emocional, problemas relacionados as atividades laborais e prejuízo nas atividade diárias sendo a segunda maior causa de dor em região de coluna vertebral, perdendo apenas para a lombalgia ${ }^{(1,3,6)}$. 
A literatura propõe que em torno de $12 \%$ a $70 \%$ dos adultos poderão ser acometidos por cervicalgia em algum momento da vida, sendo o sexo feminino o mais afetado ${ }^{(2,5,7,8)}$.

São vários os fatores associados ao surgimento da cervicalgia como esforço repetitivo, posturas inadequadas de longa permanência durante atividades laborativas, movimentos bruscos e traumas $^{(1,3,4)}$.

$\mathrm{Na}$ prática da odontologia, é percebido que os profissionais e estudantes durante as práticas na graduação executam atividades repetitivas que exigem destes posturas inadequadas por longos períodos, constituindo uma classe bastante susceptível a desenvolver lesões de origem osteomusculares. Também tem sido considerada uma profissão estressante, que acarreta problemas ligados a saúde física e psicológica ${ }^{(9-10)}$.

Alguns estudos mostram que mais de $50 \%$ desta classe profissional apresenta algum tipo de dor relacionado á alterações musculoesqueléticas provenientes do trabalho, em região do pescoço, costas, ombros e membros superiores. Estes distúrbios acabam afetando o rendimento profissional e são considerados as principais causas que levam estes indivíduos a uma aposentadoria precoce ${ }^{(11-12)}$.
Diante do exposto, entendendo que desde a graduação os indivíduos já são expostos a fatores de risco para o desenvolvimento destes distúrbios, foi proposto este trabalho que tem como objetivo verificar a prevalência de cervicalgia em acadêmicos de odontologia de um centro universitário, para que seja possível nortear o estabelecimento de estratégias para atenuar o impacto desta dor nas atividades laborativas futuras $\mathrm{e}$ atividades de vida diária.

\section{Método}

Tratou-se de um estudo observacional, analítico de caráter transversal realizado no período de janeiro a junho de 2015, em uma Clínica Escola de Odontologia de um Centro Universitário na cidade de Maceió - Alagoas.

Foram incluídos na pesquisa acadêmicos de odontologia, que no período da coleta estavam cursando $9^{\circ}$ e $10^{\circ}$ períodos, ou seja, em estágio supervisionado, de ambos os sexos e com idade superior a 18 anos. Foram excluídos os que referiam ter histórico de patologia pregressa ou diagnóstico clinico de lesão na coluna vertebral e/ou na articulação temporomandibular, com gravidez suspeita ou confirmada, 
Silva AF, Costa MAL, Soutinho RSR, Pedrosa AS 426

Prevalência de Cervicalgia em Acadêmicos de Odontologia

fibromialgicos ou com queixa de irradiação e/ou parestesia em membros superiores.

A amostra foi não aleatória, por conveniência, correspondendo ao total de alunos matriculados nos estágios durante o período da coleta.

O recrutamento dos sujeitos foi realizado mediante abordagem que ocorreu nos corredores da Clínica Escola de Odontologia, ao final das atividades do estágio, momento em que foram informados sobre a natureza da pesquisa e assinaram o Termo de Consentimento Livre e Esclarecido baseado na Resolução 466/12, do Conselho Nacional de Saúde, do Ministério da Saúde (CNS/MS).

Após a assinatura do TCLE foi aplicado o formulário de coleta de dados contendo questões relacionadas a dados sócio-demográficos (idade, sexo, estado civil), hábitos de vida como a prática de atividade física, questões sobre a existência e características da dor em região cervical e informações sobre a atuação enquanto acadêmico.

Dando sequência a pesquisa foi empregado o Índice de Incapacidade Relacionada ao Pescoço, que é um instrumento traduzido, adaptado e validado para a língua portuguesa que tem por objetivo, informar ao avaliador como a dor na região cervical tem interferido em suas atividades cotidianas $^{(13)}$. O mesmo possui dez domínios que objetivam avaliar interferência da dor quanto a: cuidados pessoais, levantamento de objetos, leitura, concentração, atividades laborais, ato de dirigir, no sono, em momentos de lazer, intensidade da dor e se sente dores de cabeça. Cada domínio é composto por seis alternativas, numeradas de zero a cinco, que descrevem graus crescentes de interferência da dor cervical sobre a realização da atividade questionada. $\mathrm{O}$ cálculo dos escores é obtido pela soma dos pontos, de 0 a 5, das 10 questões, tendo um total de no máximo 50 pontos. Considera-se o paciente sem incapacidade quando o resultado varia de 0 a 4 pontos, com incapacidade leve quando o mesmo tem uma variação de 5 a 14 pontos, de 15 a 24 pontos se classifica com incapacidade moderada, 25 a 34 incapacidade grave e incapacidade completa quando a variação é de $35-50^{(14-15)}$.

Após a coleta de dados, os resultados foram tratados através de estatística descritiva, demostrados em média e desvio padrão. $\mathrm{Na}$ análise estatística inferencial, foi utilizado o teste qui-quadrado adotando-se o nível de significância de 5\% para comparar as proporções de indivíduos com 
cervicalgia com as demais variáveis do estudo.

A pesquisa foi aprovada pelo Comitê de Ética em Pesquisa do Centro de Estudos Superiores de Maceió CESMAC, sob protocolo $\mathrm{n}^{\circ}$ 38186114.9 .0000 .0039 .

\section{Resultados}

Participaram da pesquisa 67 estudantes, dos quais $42(62,7 \%)$ são do sexo feminino e 25 (37,3\%) são do sexo masculino. Destes 16 (23,9\%) são casados ou possuem união estável e 51 $(76,1 \%)$ são solteiros. $36 \quad(53,7 \%)$ indivíduos referiram não praticar atividade física enquanto que 31 $(46,3 \%)$ relataram que sim. A média de idade encontrada foi de $24,8 \pm 4,3$.

Os estudantes incluídos estavam cursando o Estágio Curricular Supervisionado, sendo que 30 (44,7\%) eram matriculados no $9^{\circ}$ período e 37 $(55,2 \%)$ no $10^{\circ}$ período. A frequência de aulas práticas semanais era variável, onde para $29(43,28 \%)$ deles ocorria numa frequência igual ou menor que 3 vezes por semana e para $38(56,71 \%)$ ocorria numa frequência maior do que 3 vezes por semana. Quanto a área de estágio que estavam cursando no momento da coleta, $13 \quad(19,4 \%)$ estavam no estágio da pediatria, 30
(44,77\%) na área de dentística, 13 $(19,4 \%)$ no estágio de prótese e 11 $(16,41 \%)$ em cirurgia.

Quando questionados com relação à queixa de dor na região cervical, a prevalência encontrada foi de $44,8 \%$, não tendo sido observadas diferenças significativas entre a prevalência de cervicalgia e as variáveis sexo, atividade física, estado civil, período acadêmico, número de práticas semanais e área do estágio, conforme ilustrado na Tabela 1.

Considerando os participantes que relataram apresentar cervicalgia e as características da dor, quanto ao tempo de surgimento do sintoma, 9 (30\%) referiram sentir até 1 ano, $17(56,66 \%)$ indivíduos acima de 1 ano e até 3 anos e $4(13,33 \%)$ acima de 3 anos. Sobre a intensidade da dor, $14 \quad(46,6 \%)$ relataram ser leve, $16(53,3 \%)$ ser moderada e nenhum estudante relatou ser intensa.

Sobre a presença de outros sintomas relacionados à região cervical, $19(63,3 \%)$ referiram cansaço muscular, $4(13,3 \%)$ rigidez articular, $2(6,6 \%)$ perda de força e $2(6,6 \%)$ limitação de movimentos, sendo que alguns assinalaram mais de uma alternativa, confirmando a existência de múltiplos sintomas. 
Tabela 1 - Caracterização da Amostra Quanto às Variáveis Sociodemográficas, Hábitos de Vida, Dados da Prática Acadêmica

\begin{tabular}{|c|c|c|c|c|c|}
\hline \multirow[b]{3}{*}{ Variável } & \multicolumn{4}{|c|}{ Cervicalgia } & \multirow{3}{*}{$\begin{array}{l}\text { Valo } \\
\text { de } p^{*}\end{array}$} \\
\hline & \multicolumn{2}{|c|}{ Não } & \multicolumn{2}{|c|}{ Sim } & \\
\hline & $\mathrm{n}$ & $\%$ & $\mathrm{n}$ & $\%$ & \\
\hline \multicolumn{6}{|l|}{ Sexo } \\
\hline Feminino & 23 & 62,2 & 19 & 63,3 & \multirow[t]{2}{*}{0,921} \\
\hline Masculino & 14 & 37,8 & 11 & 36,7 & \\
\hline \multicolumn{6}{|c|}{ Estado Civil } \\
\hline Solteiro & 31 & 83,8 & 20 & 66,7 & \multirow[t]{2}{*}{0,102} \\
\hline Casado & 6 & 16,2 & 10 & 33,3 & \\
\hline \multicolumn{6}{|l|}{$\begin{array}{l}\text { Atividade } \\
\text { física }\end{array}$} \\
\hline Não & 17 & 45,9 & 19 & 63,3 & \multirow[t]{2}{*}{0,156} \\
\hline Sim & 20 & 54,1 & 11 & 36,7 & \\
\hline \multicolumn{6}{|l|}{$\begin{array}{l}\text { Período } \\
\text { Acadêmico }\end{array}$} \\
\hline $9^{\circ}$ & 15 & 40,5 & 15 & 50,0 & \multirow[t]{2}{*}{0,439} \\
\hline $10^{\circ}$ & 22 & 59,5 & 15 & 50,0 & \\
\hline \multicolumn{6}{|c|}{$\mathrm{N}^{\mathrm{o}}$ de práticas } \\
\hline$\leq 3$ & 13 & 35,1 & 16 & 53,3 & \multirow[t]{2}{*}{0,135} \\
\hline$>3$ & 24 & 64,9 & 14 & 46,7 & \\
\hline \multicolumn{6}{|l|}{$\begin{array}{l}\text { Área do } \\
\text { estágio }\end{array}$} \\
\hline Pediatria & 6 & 16,2 & 7 & 23,3 & \multirow[t]{4}{*}{0,076} \\
\hline Dentística & 15 & 40,5 & 15 & 50,0 & \\
\hline Prótese & 6 & 16,2 & 7 & 23,3 & \\
\hline Cirurgia & 10 & 27,0 & 1 & 3,3 & \\
\hline
\end{tabular}

Considerando os participantes que relataram apresentar cervicalgia e as características da dor, quanto ao tempo de surgimento do sintoma, 9 $(30 \%)$ referiram sentir até 1 ano, 17 (56,66\%) indivíduos acima de 1 ano e até 3 anos e $4(13,33 \%)$ acima de 3 anos. Sobre a intensidade da dor, 14 $(46,6 \%)$ relataram ser leve, $16(53,3 \%)$ ser moderada e nenhum estudante relatou ser intensa.
Sobre a presença de outros sintomas relacionados à região cervical, $19(63,3 \%)$ referiram cansaço muscular, $4(13,3 \%)$ rigidez articular, 2 $(6,6 \%)$ perda de força e $2(6,6 \%)$ limitação de movimentos, sendo que alguns assinalaram mais de uma alternativa, confirmando a existência de múltiplos sintomas.

Quanto a frequência da dor na região cervical, $2(6,6 \%)$ afirmaram 
sentir todos os dias na semana, $3(10 \%)$ mais da metade da semana, $12(40 \%)$ menos da metade da semana e 13 $(43,3 \%)$ eventualmente.

As causas citadas pelos estudantes relacionados ao surgimento da cervicalgia foram: postura para 27 (90\%) estudantes, $12 \quad(40 \%)$ consideraram os esforços repetitivos e $1(3,33 \%)$ relatou movimento brusco.

Referindo-se a interferência da dor nas atividades cotidianas, onde a mesma foi classificada através do Índice de Incapacidade Relacionada ao
Pescoço, constatou-se que $14(46,66 \%)$ estudantes não apresentavam incapacidade, 15 (50\%) incapacidade leve e apenas 1 (3,3\%) estudante incapacidade moderada.

A Tabela 2 caracteriza a amostra de estudantes com cervicalgia quanto à intensidade da dor, tempo do surgimento do sintoma, outros sintomas, freqüência, causas e classificação da intensidade da dor de acordo com o Índice de Incapacidade Relacionada ao Pescoço.

Tabela 2 - Caracterização da Amostra

\begin{tabular}{lcc}
\hline & $\mathrm{n}$ & $\%$ \\
\hline Intensidade da Dor & 14 & $46,6 \%$ \\
\hline Leve & 16 & $53,3 \%$ \\
Moderada & & \\
\hline Tempo do surgimento do sintoma & 9 & $30 \%$ \\
\hline Inferior à 1 ano & 17 & $56,6 \%$ \\
Acima de 1 ano - 3 anos & 4 & $13,3 \%$ \\
Acima de 3 anos & & \\
\hline Outros sintomas & 19 & $63,3 \%$ \\
\hline Cansaço muscular & 4 & $13,3 \%$ \\
Rigidez articular & 2 & $6,6 \%$ \\
Perda de força & 2 & $6,6 \%$ \\
Limitação de movimentos & & $6,6 \%$ \\
\hline Frequência & 2 & $10 \%$ \\
\hline Todos os dias da semana & 3 & $40 \%$ \\
Mais da metade da semana & 12 & $43,3 \%$ \\
Menos da metade da semana & 13 & \\
Eventualmente & & $90 \%$ \\
\hline Causas & 27 & $40 \%$ \\
\hline Posturas inadequadas & 12 & $3,3 \%$ \\
Esforços repetitivos & 1 & $46,6 \%$ \\
Movimentos bruscos & & $50 \%$ \\
\hline Índice de Incapacidade Relacionada ao Pescoço & $3,3 \%$ \\
\hline Sem incapacidade & 14 & \\
Com incapacidade leve & 15 & \\
Com incapacidade moderada & 1 & \\
\hline
\end{tabular}




\section{Discussão}

Muitos estudos têm relatado que problemas de origem musculoesquelética são cada vez mais freqüentes na prática da odontologia, sendo as algias relacionadas à coluna cervical e lombar as mais prevalentes $^{(2,11)}$.

As principais razões para a presença destas queixas estão relacionadas a posturas inadequadas, esforços repetitivos, movimentos bruscos e traumas, onde ao serem indagados sobre a percepção da causa da dor na cervical, $90 \%$ dos estudantes referiram ser em decorrência de posturas inadequadas durante os procedimentos odontológicos ${ }^{(16)}$. Estas posturas podem gerar sobrecarga na musculatura da cervical, levando a fadigabilidade e consequentemente a dor.

A prevalência de cervicalgia na literatura é bastante variável, com estudos que destacam entre $12 \%$ a $70 \%$, sendo o gênero feminino o mais afetado $^{(2,5,7,8)}$. O presente estudo reforça esta estatística, com uma prevalência de $44,8 \%$ nos estudantes, número já bastante alto, considerando que ainda não são profissionais e este número tende a aumentar com a prática profissional, caso não haja uma intervenção.

As mulheres também foram mais acometidas, mesmo este dado não tenha sido estatisticamente significativo, o que pode ser justificado pelo fato deste público ter maior propensão a labilidade emocional, principalmente devido às variações hormonais que ocorrem no ciclo menstrual e muitas vezes jornada dupla de trabalho e estudos, o que acaba por acarretar maior tensão da musculatura corporal. Mulheres possuem um desenvolvimento osteomuscular diferente dos homens com um número reduzido de fibras musculares o que reduz a capacidade de armazenar e converter glicogênio em energia útil ${ }^{(17)}$.

Estudos que avaliam prevalência de cervicalgia em cirurgiões dentistas, também não houve associação entre a variável estado civil e presença de cervicalgia, sendo a maior parte dos entrevistados $(70 \%)$ casados, o que pode ser justificado pelo fato da amostra ter sido composta por profissionais, enquanto que na presente pesquisa a maioria $(76,1 \%)$ eram solteiros, o que é esperado para uma população universitária ${ }^{(2)}$.

Quando indagados sobre a prática de atividade física 36 indivíduos 
referiram não praticar e destes, 19 relataram dor na região cervical. $\mathrm{O}$ estudo realizado por Siqueira e outros autores $^{(10)}$, mostrou que a maioria dos entrevistados $(55,8 \%)$ também não praticava atividade física, e embora este dado não tenha apresentado correlação com a dor, assim como na presente pesquisa, supõe-se que a falta de condicionamento físico pode levar a fraqueza muscular e consequentemente a sobrecarga de várias regiões do corpo durante a prática da odontologia.

Uma das áreas de estágio e principal campo de atuação da odontologia é a dentística, que é uma especialidade, que previne, diagnostica e restaura a função dental, cuidando também da estética e da função fisiológica dos dentes ${ }^{(18)}$. Nesta área, a principal demanda de atendimento é o tratamento de lesões cariogênicas que necessitam de cuidados tais como restaurações, que consiste na retirada de tecido e inserção de um material restaurador.

A maioria dos acadêmicos entrevistados relatou estar no estágio referente a esta área de atuação e 50\% deles referiam ter cervicalgia. Sabe-se que durante a prática nesta área, eles necessitam de movimentos finos e precisos, onde permanecem com a cervical em posturas fixas, aumentando o nível de tensão nesta região, o que pode ter uma forte relação com o surgimento da queixa. Estudos reforçam que os procedimentos de clínica geral são os que apresentaram maior atividade eletromiográfica dos músculos posturais analisados, o que pode ser justificado pelo fato dos procedimentos dessa especialidade exigirem maior atividade dos músculos por serem mais complexos ${ }^{(12)}$.

Pesquisas apontas que dor musculoesquelética em estudantes de odontologia, encontrou que estes já apresentavam intensidade de dor moderada e intensa, sem diferenças significativas com relação ao sexo ${ }^{(10)}$. O presente estudo observou que os estudantes relataram dor leve a moderada e nenhum deles relatou dor intensa, o que talvez possa ser atribuído às características individuais como também a organização da prática supervisionada de cada instituição.

Quanto a presença de outros sintomas relacionados à região cervical que não a dor, $19(63,3 \%)$ referiram cansaço muscular, 4 (13,3\%) rigidez articular, $2(6,6 \%)$ perda de força e 2 $(6,6 \%)$ limitação de movimentos. Um estudo feito em cirurgiões dentistas que outras queixas são comuns nesta prática citando tensão muscular, impaciência, formigamento, fadiga 
constante, queimação, tremores, fraqueza e palpitações ${ }^{(16)}$.

Quanto ao tempo de aparecimento do sintoma, é possível inferir que o tempo de prática clínica possa ter relação com o surgimento da dor já que $30 \%$ dos entrevistados referiram apresenta-la em um tempo até 1 ano, 56,6\% acima de 1 e até 3 anos e superior a 3 anos 13,3\%. Analisando a matriz curricular do curso de odontologia da instituição pesquisada, observa-se que as práticas se iniciam no segundo ano do curso e se intensificam no 5 ano, período destinado ao estágio curricular supervisionado.

Em relação à interferência da dor nas atividades cotidianas, classificada pelo Índice de Incapacidade Relacionada ao Pescoço, no atual estudo constatou-se que 14 indivíduos não tiveram incapacidade, 15 indivíduos apresentaram incapacidade leve e apenas 1 indivíduo incapacidade moderada, supondo-se que o tempo de prática odontológica possa ter relação com o grau de interferência em atividades como por exemplo cuidados pessoais. Como ainda são estudantes e não vivenciam a prática como os profissionais e nem apresentam outros fatores como o peso da responsabilidade profissional, isso pode

ter justificado uma menor interferência nestas atividades na população estudada.

\section{Conclusão}

Foi possível observar que a prevalência de cervicalgia nos acadêmicos de odontologia enquadra-se nos valores propostos pela literatura, necessitando de uma atenção especial para este público que ainda será exposto a fatores de risco durante a prática profissional.

Desta forma o presente estudo se mostra relevante para a população em questão, instigando a necessidade da realização de novas pesquisa e assim aprofundar o conhecimento sobre as causas que influenciam no aparecimento da dor, objetivando elimina-las, permitindo o norteio de medidas preventivas, com o propósito de melhorar as condições laborais deste público, reduzindo assim o número de acadêmicos que possam progredir para a vida profissional, apresentando tal distúrbio.

\section{Referências}

Revist. Port.: Saúde e Sociedade. 2017; 2(2):422-434. 
1. Sobral MKM, Silva PG, Vieira RAG, Siqueira RS . A efetividade da terapia de liberação posicional (TLP) em pacientes com cervicalgia. Fisioterapia (Curitiba) [periódico na Internet]. 2010 [citado 2017 Jan. 03]; 23(4): 513-21. Disponível em: http://www.scielo.br/scielo.php?script $=$ sci_arttext\&pid $=$ S0103-515020100 00400002 .

2. Melo RS, Pereira TR. Prevalência de algias vertebrais em cirurgiões dentistas. Cirurgiões Dentistas (Buenos Aires) [periódico na Internet]. 2011 [citado 2017 Jan. 05];15(157):1. Disponível em: http://www.efdeport es.com/efd157/algias-vertebrais-em-cir urgioes-dentistas.htm.

3. Silva RMV, Lima MS, Costa FH, Silva AC. Efeito da quiropraxia em pacientes com cervicalgia: revisão sistemática. (São Paulo) [periódico na Internet]. 2012 [citado 2017 Jan. 07];13(1):71-4. Disponível em: http://www.scielo.br/scielo.php?script $=$ sci_arttext\&pid $=\mathrm{S} 1806-0013201200$ 0100013 .

4. Soares JC, Weber P, Trevisan ME, Trevisan CM, Mota CB, Rossi AG. Influência da dor no controle postural de mulheres com dor cervical. (Rio Grande do Sul) [periódico na Internet]. 2013 [citado 2017 Jan. 07]; 15(3):37181. Disponível em: http://www.scielo .br/scielo.php?pid $=$ S1980-0037201300 0300011\&script=sci_abstract\&tlng=pt.

5. Soares JC, Weber P, Trevisan ME, Trevisan CM, Mota CB, Rossi AG. Correlação entre postura da cabeça, intensidade da dor e índice de incapacidade cervical em mulheres com queixa de dor cervical. Fisioterapia (São Paulo) [periódico na Internet]. 2012 [citado 2017 Jan. 07]; 19(1):68-72. Disponível em: http://www.scielo.br/scielo.php?script $=$ sci_arttext\&pid=S1809-29502012000 100013 .
6. Borges MC, Borges CS, Silva AGJ, Castellano LRC, Cardoso FAG. Avaliação da qualidade de vida e do tratamento fisioterapêutico em pacientes com cervicalgia crônica. Fisioterapia (Curitiba) [periódico na Internet]. 2013 [citado 2017 Jan. 15]; 26(4): 873-881. Disponível em: http://www.scielo.br/scielo.php?script $=$ sci_arttext\&pid=S0103-5150201300 0400016.

7. Delfino PD, Rampim DB, Alfieri Fm, Tomikawa LCO, Fadel G, Stump PRNAG, et al. Cervicalgia: Reabilitação. [periódico na Internet]. 2012 [citado 2017 Jan. 15]; 19(2): 7381. Disponível em: http://www.actafi siatrica.org.br/detalhe_artigo.asp?id=1 68.

8. Gadin AMV, Felipe FAA. Estudo comparativo entre dois protocolos de tratamento Flor de Liz e Método Canal Unitário em algias cervicais. [periódico na Internet]. 2013 [citado 2017 Jan. 17]; 14(4):394-4. Disponível em: http://www.scielo.br/scielo.php?pid=S 1806-00132013000400011\&script $=$ sci abstract\&tlng $=$ pt.

9. Maehler P. Estudos das sobrecargas posturais em acadêmicos de odontologia da Universidade Estadual do Oeste do Paraná- UNIOESTECascavel. [monografia]; 2003. Disponível em: http://www.unioeste.b r/projetos/elrf/monografias/2003/mono /14.pdf.

10. Siqueira GR, Silva AM, Vieira RAG, Silva RB. Dores musculoesqueléticas em estudantes de odontologia. Medicina do Trabalho (Goias) [periódico na Internet]. 2010 [citado 2017 Fev. 02]; 23(2):150-9. Disponível em: http://www.anamt.org .br/site/upload_arquivos/revista_brasile ira_de_medicina_do_trabalho_-_volu me_13_n\%C2\%BA_1_157201516121 7055475.pdf 
11. Teixeira VP, Schimidt AG, Lima MC. Prevalência de sintomas musculoesqueléticos em cirurgiões dentistas na zona norte de São Paulo. [periódico na Internet]. 2013 [citado 2017 Fev. 04]; 31(2):197-00. Disponível em: https://www.unip.br/ comunicacao/publicacoes/ics/edicoes/2 013/02_abr-jun/V31_n2_2013_p197a 200.pdf.

12.Casarin CAS, Caria PHF. Comportamento muscular durante diferentes práticas odontológicas. Cirurgia Dentista (São Paulo) [periódico na Internet]. 2008 [citado 2017 Fev. 07]; 11(2): 64-0. Disponível em: https://www.bds.ict.unesp.br/in dex.php/cob/article/download/465/388.

13. Cook C, Richardson JK, Braga L, Menezes A, Soler X, Kume P, Zaninelli M, Socolows F, Pietrobon R. Cross-Cultural Adaptation and Validation of the Brazilian Portuguese Version of the Neck Disability Index and Neck Pain and Disability Scale. Spine. (Philadelphia) [internet newspaper]. 2006 [quoted 2017 Jun. 14]; 31(14): 1621-27. Available in: https://www.ncbi.nlm.nih.gov/pubmed/ 16778699.

14. Costa DRA. Avaliação da incapacidade cervical e sua associação com dor miofacial mastigatória e hipersensibilidade mecânica generalizada em indivíduos com disfunção temporomandibular. Tese [Universidade Federal de Sergipe]; 2014. Disponível em: https://bdtd.ufs. br/handle/tede/905.
15. Pereira M. Contribuição para a adaptação cultural do Neck Disability Index e caraterização da prática de fisioterapia em pacientes com Dor Crónica Cervical. Tese [Instituto Politécnico de Sétubal]; 2012. Disponível em: https://run.unl.pt/bitstream/10362/9108 /1/Pereira\%20Marta\%20TM\%202012. pdf.

16. Pereira ACVF, Graça CS. Prevalência de dor musculoesquelética relacionada ao trabalho e cirurgiõesdentistas atuantes na rede do sistema único de saúde (SUS) no município de camaçari-BA. Monografia [FAISA]; 2012. Disponível em: https://run.unl.pt/bitstream/10362/9108 /1/Pereira\%20Marta\%20TM\%202012. pdf.

17. Kotliarenko A, Michael-Crosato E, Biazevic MGH, Crosato E, Silva PR. Distúrbios osteomusculares e fatores associados em cirurgiões dentistas do meio oeste do estado de Santa Catarina. Odontologia (São Paulo) [periódico na Internet]. 2009 [citado 2017 Fev. 15]; 24(2):173-179. Disponível em: http://revistaseletron icas.pucrs.br/ojs/index.php/fo/article/vi ewFile/4243/3999.

18. Conceição EN, Leite CV. Atuação em Dentística Saúde e Estética. Artmed; 2007. 